\title{
Effects of Sleep Deprivation on Serotonergic Neuronal Activity in the Dorsal Raphe Nucleus of the Freely Moving Cat
}

\author{
James P. Gardner, Ph.D., Casimir A. Fornal, Ph.D., and Barry L. Jacobs, Ph.D.
}

Total sleep deprivation (TSD) for one or more nights produces a rapid antidepressant response in humans. Since most pharmacological treatments for depression increase brain serotonin neurotransmission, the purpose of the present study was to determine whether TSD increases the activity of serotonergic neurons in the dorsal raphe nucleus (DRN) in cats. Cats were prevented from sleeping by the experimenter, who monitored the behavioral state of each animal on a polygraph. Firing rates during quiet waking $(Q W)$ and active waking $(A W)$ were obtained throughout a 24 - $h$ sleep deprivation period and subsequent 6- $h$ recovery period. During the experiments, unit activity was also recorded during exposure to loud white noise, which elicited strong behavioral arousal. The inhibitory response of serotonergic DRN neurons to systemic administration of the selective 5-HT ${ }_{1 A}$ agonist 8-hydroxy-2-(di-n-propylamino)tetralin (8-OH-DPAT) was determined before and after TSD to assess possible changes in $5-H T_{1 A}$ autoreceptor sensitivity.
TSD increased mean firing rates by as much as $18 \%$ during both $A W$ and white noise exposure. Maximal effects were observed after $15 \mathrm{~h}$ of TSD for $A W$, and after $18 \mathrm{~h}$ for white noise. QW firing rates also tended to be elevated throughout TSD. Firing rates for all conditions during the recovery period were not significantly different from baseline. The neuronal inhibition produced by 8-OH-DPAT was significantly diminished after TSD. Overall, these results indicate that TSD increases the firing rate of serotonergic $D R N$ neurons during $A W$ and arousal. This effect may be attributable to a decrease in the sensitivity of $5-\mathrm{HT}_{1 \mathrm{~A}}$ autoreceptors. These findings are consistent with the hypothesis that TSD exerts its antidepressant action, at least in part, through an activation of brain serotonergic neurons. [Neuropsychopharmacology 17:72-81, 1997] (C) 1997 American College of Neuropsychopharmacology. Published by Elsevier Science Inc.
KEY WORDS: Serotonin; Sleep deprivation; Depression; Antidepressant response; Dorsal raphe nucleus; $5-H T_{1 A}$ autoreceptors

Most current pharmacological treatments for depression affect the brain serotonergic system (for review see Blier and de Montigny 1994). Numerous in vivo and in vitro studies have shown that various antidepressant agents potentiate the action of serotonin $(5-\mathrm{HT})$ at cen-

From the Program in Neuroscience, Department of Psychology, Princeton University, Princeton, New Jersey.

Address correspondence to: Dr. Barry L. Jacobs, Program in Neuroscience, Green Hall, Princeton University, Princeton, NJ 08544.

Received October 29, 1996; revised February 7, 1997; accepted February 17, 1997. tral synapses through a variety of mechanisms, including inhibiting 5-HT reuptake or preventing its metabolic degradation or by increasing the synthesis and release of 5-HT from nerve terminals.

A nonpharmacological intervention that alleviates depression is total sleep deprivation (TSD) for one or more nights. This treatment is effective in approximately $60 \%$ of all depressed patients and in about $70 \%$ of those patients diagnosed with "endogenous" depression (for review, see Leibenluft and Wehr 1992; Wu and Bunney 1990). These response rates are comparable to those seen with various antidepressant drugs. A striking feature of sleep deprivation is that it produces a rapid improvement in mood, in contrast to pharmaco- 
logical treatments, which typically require several weeks to produce a therapeutic response. However, TSD is of limited clinical usefulness since its antidepressant action quickly dissipates after the patient has had some sleep. Although the effects of sleep deprivation on mood are short-lived, this manipulation is noteworthy because it demonstrates that a rapid antidepressant response is possible. Thus, a greater understanding of the neurochemical mechanisms that underlie this antidepressant action of TSD might aid in the development of faster acting antidepressant therapies. Despite the fact that most pharmacological treatments for depression affect the brain serotonergic system, the effect of TSD on the activity of serotonergic neurons has not been previously studied in conscious animals.

Further evidence suggesting that brain serotonergic neurons may be involved in the antidepressant action of sleep deprivation is the fact that their activity varies dramatically across the sleep-wake cycle (Fornal and Jacobs 1988). Serotonergic neurons throughout the brainstem fire fastest during periods of behavioral arousal, slower during drowsiness and slow-wave sleep, and typically fall silent during REM sleep (Fornal and Jacobs 1988). The close relationship between serotonergic neuronal activity and behavioral state suggests that the activity of this neurochemical system may be altered by behavioral manipulations such as sleep deprivation.

Somatodendritic 5- $\mathrm{HT}_{1 \mathrm{~A}}$ autoreceptors are hypothesized to play a crucial role in the therapeutic actions of various antidepressant drugs, including the monoamine oxidase inhibitors (MAOIs) and the selective serotonin reuptake inhibitors (SSRIs) (Blier and de Montigny 1994). Recent studies have shown that these drugs preferentially increase the concentration of extracellular 5-HT in the midbrain raphe nuclei (Ferrer and Artigas 1994; Invernizzi et al. 1992), where the cell bodies of serotonergic neurons are located. This leads to an inhibition of serotonergic neuronal activity through increased activation of $5-\mathrm{HT}_{1 \mathrm{~A}}$ autoreceptors. The decrease in activity limits the ability of these drugs to increase 5-HT levels in the forebrain (e.g., frontal cortex) and may thus explain the failure of these agents to produce an immediate therapeutic effect in the clinic. Blier and de Montigny (1994) have hypothesized that, in patients receiving antidepressant medication, the sustained elevation in 5-HT levels desensitizes or downregulates somatodendritic 5- $\mathrm{HT}_{1 \mathrm{~A}}$ autoreceptors after several weeks. As a result, autoreceptor-mediated feedback inhibition is diminished and serotonergic neurons resume firing despite continued drug administration. This restoration of serotonergic cell firing during sustained inhibition of 5-HT reuptake or degradation is thought to increase net 5-HT neurotransmission and ultimately produce an antidepressant effect.

Since the various treatments for depression share in common the ability to enhance central 5-HT neu- rotransmission, we hypothesized that the antidepressant effect of TSD may likewise be due to increased serotonergic neurotransmission. Sleep deprivation would be expected to produce an overall increase in serotonergic cell firing and presumably stimulation of postsynaptic 5-HT receptors, by preventing the normal reductions in neuronal activity that accompany sleep. In addition, the sustained firing of serotonergic neurons during prolonged wakefulness should result in the tonic activation of somatodendritic $5-\mathrm{HT}_{1 \mathrm{~A}}$ autoreceptors, which may cause desensitization of this receptor and lead to an increase in the firing rate of serotonergic neurons. Recently, we have shown that $5-\mathrm{HT}_{1 \mathrm{~A}}$ autoreceptors exert a tonic inhibitory influence on the activity of serotonergic neurons in freely moving cats during wakefulness, but not during sleep (Fornal et al. 1994, 1996).

The present study examined the effects of TSD and subsequent recovery on the single-unit activity of serotonergic neurons in freely moving cats. In addition, the responses of serotonergic neurons to a strong behaviorally arousing stimulus (white noise), and to phasic sensory stimulation (clicks) were determined during the course of these experiments. We also measured the inhibitory response of these neurons to the selective $5-\mathrm{HT}_{1 \mathrm{~A}}$ agonist 8-hydroxy-2-(di-n-propylamino)tetralin (8-OH-DPAT) before and after TSD. This was done as an initial assessment of possible changes in the sensitivity of somatodendritic $5-\mathrm{HT}_{1 \mathrm{~A}}$ autoreceptors produced by sleep deprivation. The serotonergic cell group selected for study was the dorsal raphe nucleus (DRN), since these neurons project extensively throughout the forebrain and have been implicated in several affective disorders.

\section{METHODS}

\section{Animals}

Nine male cats, weighing between 2.5 and $5.0 \mathrm{~kg}$, were used in these experiments. They were housed individually in a temperature-controlled $\left(22 \pm 1^{\circ} \mathrm{C}\right)$ and lightcontrolled (lights on from 7:00 A.M. to 9:00 P.M.) room and had free access to food (commercial diet) and water. All cats were cared for and used in strict accordance to the PHS Guide for the Care and Use of Laboratory Animals. All procedures were reviewed and approved by the Institutional Animal Care and Use Committee of Princeton University.

\section{Surgical Procedure and Postoperative Care}

Cats were anesthetized with pentobarbital sodium (40 $\mathrm{mg} / \mathrm{kg}, \mathrm{IP}$ ) and placed in a stereotaxic instrument. A microdrive, consisting of two inner stainless steel cannulas ( 23 gauge) separated by $1 \mathrm{~mm}$, which could be lowered through two outer guide cannulas (19 gauge), 
was stereotaxically implanted above the DRN at an angle of $45^{\circ}$ posterior to the vertical. Microelectrode bundles were then lowered through both cannulas so that their tips were positioned $1 \mathrm{~mm}$ above the DRN. Stereotaxic coordinates for the anterior bundle were posterior $1.5 \mathrm{~mm}$, lateral $0 \mathrm{~mm}$, and horizontal $+1.0 \mathrm{~mm}$. Each bundle consisted of three $32 \mu \mathrm{m}$ and three $64 \mu \mathrm{m}$ diameter Formvar-insulated nichrome wires. Additionally, electrodes for recording the cortical electroencephalogram (EEG), electrooculogram (EOG), and the nuchal electromyogram (EMG) were implanted. The leads from all electrodes were soldered to a 25-pin connector, and the entire apparatus was anchored to the skull with dental acrylic.

Cats were treated postoperatively, twice daily, for up to 10 days with ampicillin $(20 \mathrm{mg} / \mathrm{kg}$, IM or $250 \mathrm{mg}$, $\mathrm{PO}$ ). A topical antibiotic powder (nitrofurazone) was regularly applied to the implant incision. Cats were allowed to recover for at least 2 weeks before experiments were initiated. All cats were determined by veterinary examination to be in excellent health when these experiments were conducted.

\section{Electrophysiological Recordings}

Electrical potentials were recorded from each cat using a counterweighted low-noise cable system and 24-channel slip ring assembly. Microelectrode potentials were amplified (Grass 7P511 ac preamp), filtered (band-pass $0.1-3.0 \mathrm{kHz}$ ), and monitored continuously on a storage oscilloscope. A second microelectrode served as an indifferent electrode. Single-unit activity was separated from background noise by means of a time-amplitude window discriminator (Bak Electronics, Model DIS-1). The acceptance pulse output of the window discriminator was used to produce an on-line record of cell discharge through both a speaker and an electronic counter, and on a polygraph (Grass model 7C). During most experiments, individual action potentials were digitized (12 bit analog-to-digital converter, $20 \mathrm{kHz}$ sampling rate) and stored on a computer for later analysis. Cortical EEG, EOG, and EMG potentials were amplified (Grass 7P5 or 7P511 ac preamp), band-pass filtered (EEG, 1-35 Hz; EOG, 1-35 Hz; EMG, 30-90 Hz), and recorded continuously on the polygraph.

All experimental trials were conducted in an electrically shielded, sound-attenuating chamber $(65 \times 65 \times$ $95 \mathrm{~cm}$ high) with a transparent Plexiglas door, which allowed for remote television monitoring of the animals. Each animal was habituated daily to the recording chamber over a 2-week postoperative period. After habituation, the microdrive was advanced through the DRN in discrete steps $(\sim 80 \mu \mathrm{m})$ until stable single-unit recordings characteristic of serotonergic neurons were encountered. Only recordings that displayed a signalto-noise ratio of at least 3:1 were used for data collec- tion. To ensure that recordings were made from the same cell, the action potential waveform of the neuron was monitored continuously throughout the experiments by a computer (Figure 1).

\section{Neuronal Identification}

During quiet waking (defined below), individual neurons in the DRN were initially identified on-line as serotonergic according to previously established criteria (Fornal and Jacobs 1988): (1) slow and highly regular discharge activity ( $\sim 1-5$ spikes/s); (2) biphasic action potentials of relatively long duration (>2 ms); (3) marked suppression of activity during rapid-eye-movement (REM) sleep, and in response to systemic administration of the $5-\mathrm{HT}_{1 \mathrm{~A}}$ agonist 8-OH-DPAT; and (4) histological localization of recording sites to the region of the DRN.

\section{Sleep Deprivation}

After the isolation of a stable serotonergic unit recording and the collection of baseline data, a 24-h period of TSD was initiated. Cats were prevented from sleeping by the experimenter, who monitored the behavioral state of the animal on a polygraph and aroused the animal at the onset of each sleep episode. In addition to monitoring the polygraph, the experimenter was alerted whenever the cat began showing signs of drowsiness by an alarm activated each time the cortical EEG amplitude exceeded $100 \mu \mathrm{V}$. Initially, a simple tap on the door of the recording booth was sufficient to arouse the cat. However, with increased sleep deprivation, it became more difficult to prevent the cat from sleeping, and the experimenter had to physically interact with the animal, by playing with or petting the cat, to induce arousal. No aversive or noxious stimuli were used in this study.

The sleep deprivation experiments began at different times of the day (9:00 A.M. to 9:30 P.M), with most experiments starting in the middle of the afternoon. The sleep deprivation experiments were conducted under constant illumination. Lights were dimmed during the recovery period, except when data were being collected. Each cat was allowed to recover for at least 2 weeks between successive sleep deprivation sessions, and no cat was sleep deprived on more than four occasions. Cats housed under laboratory conditions typically sleep approximately $16 \mathrm{~h}$ per day, including several hours spent in drowsiness, with sleep fairly evenly distributed across the light/dark cycle (Tobler and Scherschlicht 1990).

\section{Behavioral State Assessment and Data Collection}

For the purpose of the present study, wakefulness was divided into two general categories based on polygraphic criteria and visual observation of the animal: 
Quiet waking (QW), characterized by a predominantly low-voltage, fast (desynchronized) EEG, and absence of both large $(\geq 400 \mu \mathrm{V})$ eye movement potentials and phasic EMG bursts, and a lack of gross body movements (e.g., maintenance of a sitting or lying posture with head up); active waking (AW), characterized by a desynchronized EEG, phasic EMG bursts superimposed on high-amplitude tonic EMG, frequent ( $\geq 20$ / min) and large eye movement potentials and occurrence of gross body movement (e.g., walking). Unit activity samples during both $\mathrm{QW}$ and AW were obtained during the baseline recording period and at 3-h intervals throughout the sleep deprivation and recovery periods. Each sample consisted of 2-5 min of activity, divided into 10-s blocks, from which mean firing rates were calculated. In many instances, it was necessary for the experimenter to continuously interact with the cat to maintain an AW state.

\section{Exposure to White Noise}

Loud white noise was used to produce an alert behavioral state in the cats. After collection of click data (see below), cats were exposed to $100 \mathrm{~dB}$ white noise for 30 $\mathrm{s}$. The noise stimulus was presented before sleep deprivation (baseline), at 6-h intervals throughout the sleep deprivation, and after $6 \mathrm{~h}$ of recovery. The discharge rate of each neuron during white noise was determined and expressed as a percentage of the baseline response.

\section{Exposure to Repeated Click}

Cats were exposed to a series of 60 clicks $(110 \mathrm{~dB}, 1 \mathrm{~ms}$ duration, $1 \mathrm{kHz}$ ), delivered at a rate of one click every 5 $\mathrm{s}$. The clicks were presented before sleep deprivation (baseline), at 6-h intervals throughout the sleep deprivation period, and after $6 \mathrm{~h}$ of recovery. A peri-stimulus time histogram (PSTH) of unit activity was generated using software from DataWave Technologies (Thornton, $\mathrm{CO}$ ). PSTHs were analyzed on an individual cell basis.

\section{8-OH-DPAT Administration}

The response of serotonergic neurons to systemic administration of the selective $5-\mathrm{HT}_{1 \mathrm{~A}}$ agonist $8-\mathrm{OH}-$ DPAT was determined before and after TSD. The challenge dose ( 2 to $50 \mu \mathrm{g} / \mathrm{kg}$ ) of 8-OH-DPAT used for each cell produced a marked, but incomplete suppression of neuronal activity. Complete recovery of firing was observed within $1 \mathrm{~h}$ of injection. After obtaining baseline unit activity, ( \pm ) 8-OH-DPAT hydrobromide (Research Biochemicals International, Natick, MA), dissolved in

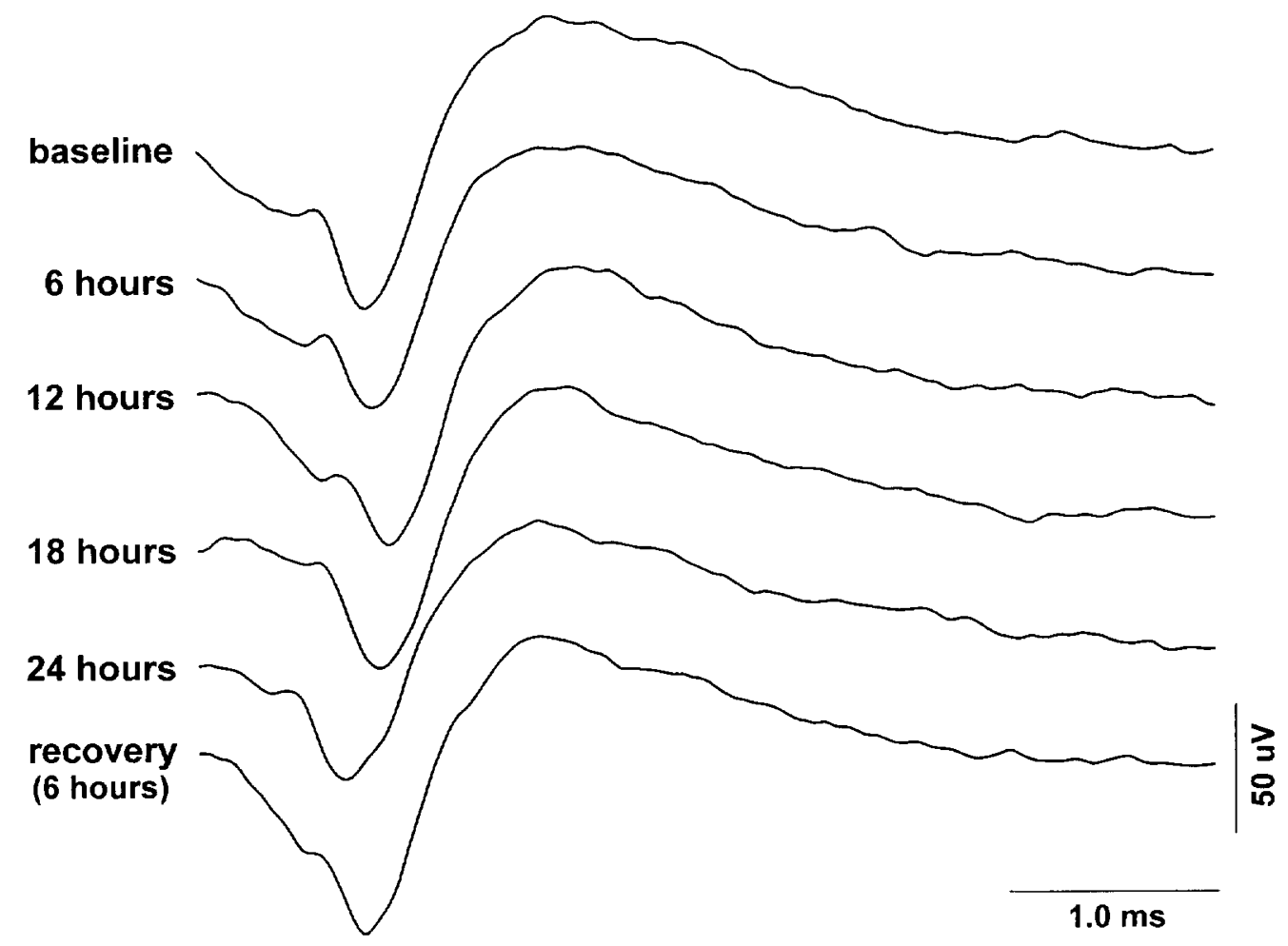

Figure 1. Extracellular action potentials of one serotonergic DRN neuron recorded at different times during the course of sleep deprivation and recovery. The action potential waveform of individual neurons was continuously monitored during these experiments to ensure that the same cell was recorded. Note the relatively long duration of the action potential, which is characteristic of serotonergic neurons, and the consistency of the action potential waveform throughout the experiment. 
sterile normal saline, was injected subcutaneously into the back of the neck. Unit activity was continuously recorded throughout the entire drug response. After neuronal activity returned to baseline levels, cats were allowed a minimum of $4 \mathrm{~h}$ before starting TSD to ensure a complete washout of 8-OH-DPAT, which might have otherwise influenced the activity of serotonergic neurons during the sleep deprivation period. 8-OH-DPAT was not administered in every experiment because the drug could potentially interfere with the collection of data during the recovery period. Changes in firing rates in response to 8-OH-DPAT were determined by comparing the discharge rate taken over a 1-min period during the time of peak drug effect to the rate obtained during a 1-min period immediately before the injection. Data were expressed as a percentage of baseline and as the absolute rate (i.e., spikes/s) decrease from baseline.

\section{Histology}

Animals were deeply anesthetized with pentobarbital sodium $(50 \mathrm{mg} / \mathrm{kg}, \mathrm{IP})$ and direct anodal current was passed through the recording electrode $(20 \mu \mathrm{A}$ for $40 \mathrm{~s})$ at sites from which acceptable units were recorded. Cats were then perfused intracardially with physiological saline followed by $10 \%$ formalin, and then $5 \%$ potassium ferrocyanide in formalin to produce a Prussian blue reaction. Frozen sections $(50 \mu \mathrm{m})$ were cut through the midbrain region, mounted on slides, and stained with neutral red.

\section{Statistical Analysis}

All data are expressed as mean \pm SEM, unless otherwise stated. Group firing rate data obtained during the sleep deprivation and recovery periods were analyzed using a repeated measures analysis of variance (ANOVA). Simple effects were subsequently analyzed using a Student's Newman-Keuls (SNK) test. Individual cell responses to sleep deprivation were analyzed using a simple ANOVA and subsequent SNK test. AW or QW firing rates were considered to be increased or decreased by sleep deprivation if at least two consecutive timepoints were significantly different from baseline. The 8-OH-DPAT data obtained before and after sleep deprivation were analyzed using a paired $t$-test. Multiple regression analysis was used to determine which factors predicted the response of serotonergic neurons to sleep deprivation. The dependent variable used in these analyses was the peak increase (percent) in AW firing rate produced by sleep deprivation. Only cells that were recorded for at least $18 \mathrm{~h}$ of sleep deprivation were included in the multiple regression analysis. A probability value $\leq .05$ was taken as statistically significant.

To analyze the effects of repeated click exposure, unit activity was divided into five consecutive $100 \mathrm{~ms}$ bins, beginning $100 \mathrm{~ms}$ before the stimulus and ending $400 \mathrm{~ms}$ after the stimulus. For each timepoint during the experiment, the 60 click trials were divided into six blocks of 10 consecutive stimulus presentations and the mean ( \pm SEM) number of spikes during each $100 \mathrm{~ms}$ bin was calculated and used for data analysis by a factorial ANOVA (TSD/recovery timepoint $\times$ bin). Post hoc analysis of the main effects and interactions was performed using an SNK analysis.

\section{RESULTS}

Data were obtained from 20 neurons recorded in nine cats. All neurons were classified as serotonergic based on previously established criteria (Fornal and Jacobs 1988). The mean duration of the action potential of these neurons was $2.4 \pm 0.2 \mathrm{~ms}$. During $\mathrm{QW}$, all neurons exhibited a relatively slow (range $=1.45$ to 7.01 spikes/s) and regular discharge pattern. Mean firing rates were $3.24 \pm 0.33$ spikes/s during AW and $3.04 \pm$ 0.33 spikes/s during QW. Unit activity steadily declined during slow-wave sleep, reaching its lowest level during REM sleep. Of the 20 neurons studied, 11 were completely silent during REM sleep. The mean percentage decrease in firing rate from QW to REM sleep was $84 \pm 5 \%(n=20$ cells). In addition, all cells tested with systemic $8-\mathrm{OH}-\mathrm{DPAT}$ displayed a marked suppression of unit activity.

Sixteen neurons were recorded for the entire 24-h sleep deprivation period. Twelve of these were subsequently recorded throughout the 6-h recovery period. The four cells that were not followed for the entire deprivation period were lost due to signal degradation after $9,15,18$, and $21 \mathrm{~h}$ of TSD.

\section{Effects on AW and QW Firing Rates}

The effect of TSD and subsequent recovery on the activity of serotonergic DRN neurons during $\mathrm{AW}$ is shown in Figure 2. A one-way ANOVA revealed a significant effect of time on unit activity $(p<.01)$. Subsequent analysis showed that sleep deprivation produced a significant increase in neuronal activity that began at $6 \mathrm{~h}$ and continued to the end of the deprivation period. The largest increase in mean firing rate produced by sleep deprivation was $18.3 \pm 4.8 \%(n=18$ cells $)$ and occurred at $15 \mathrm{~h}$. AW firing rates during the recovery period were not significantly different from baseline. During the recovery period, all cats fell asleep and displayed long episodes of deep slow-wave sleep and REM sleep. Since a substantial number of data points were missing from the recovery period, another ANOVA was performed on just the TSD data. This analysis also revealed a significant effect of TSD on neuronal activity $(p<.01)$. 


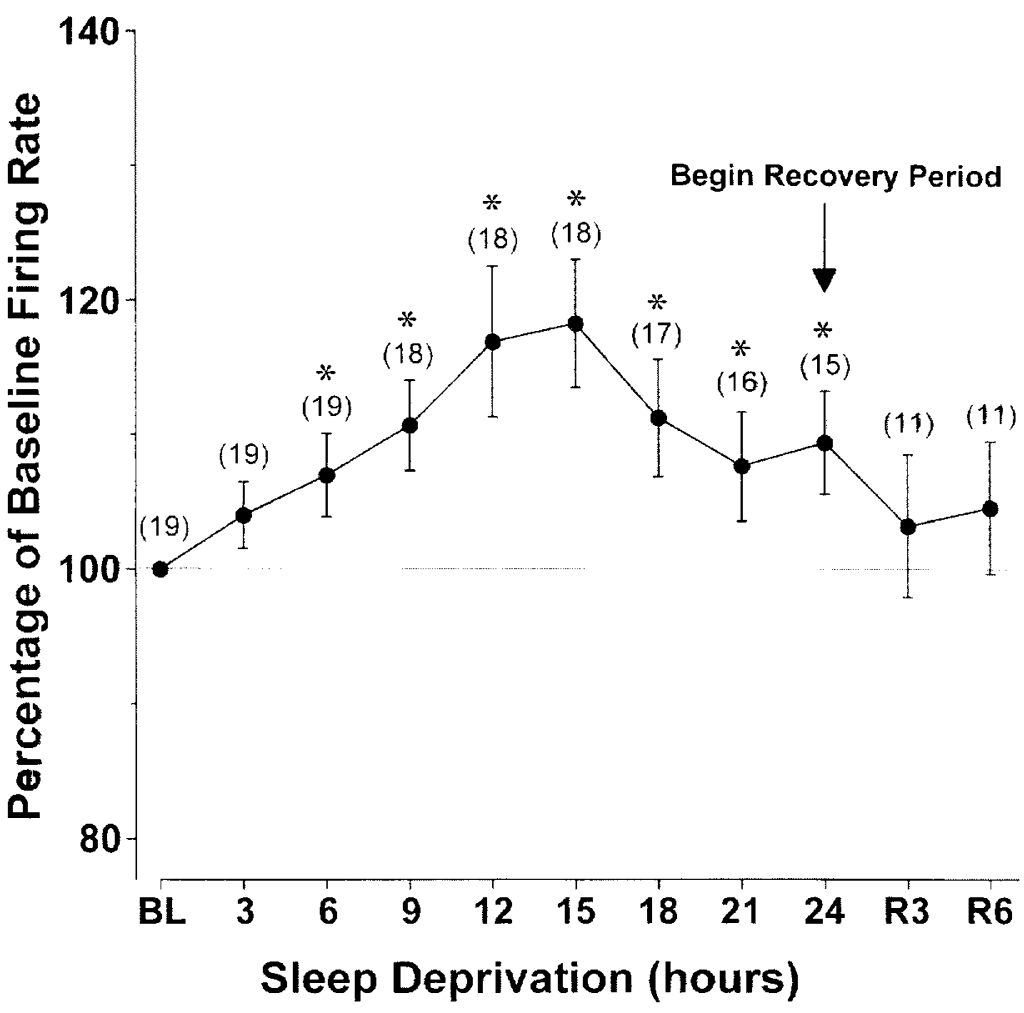

Figure 2. Effect of total sleep deprivation (TSD) and recovery on the activity of serotonergic DRN neurons during active waking (AW). Values are means \pm SEM; $n=$ number in parentheses. $\mathrm{BL}=$ baseline. The mean $\mathrm{AW}$ firing rate of these neurons prior to sleep deprivation was $3.24 \pm 0.33$ spikes $/ \mathrm{s}$ ( $n=19$ cells). Unit activity was significantly increased during the deprivation period, but not during the subsequent recovery period. ${ }^{*} p<.05$ vs. baseline.

Figure 3 displays the activity of individual neurons during AW across the TSD and recovery periods. Of the 19 neurons for which AW data were obtained during TSD, 12 showed a significant increase in firing rate, three showed a significant decrease, and the remaining four cells showed no significant change in activity. The amount of TSD required to produce the peak increase in firing rate for individual neurons ranged from 6 to $24 \mathrm{~h}$.

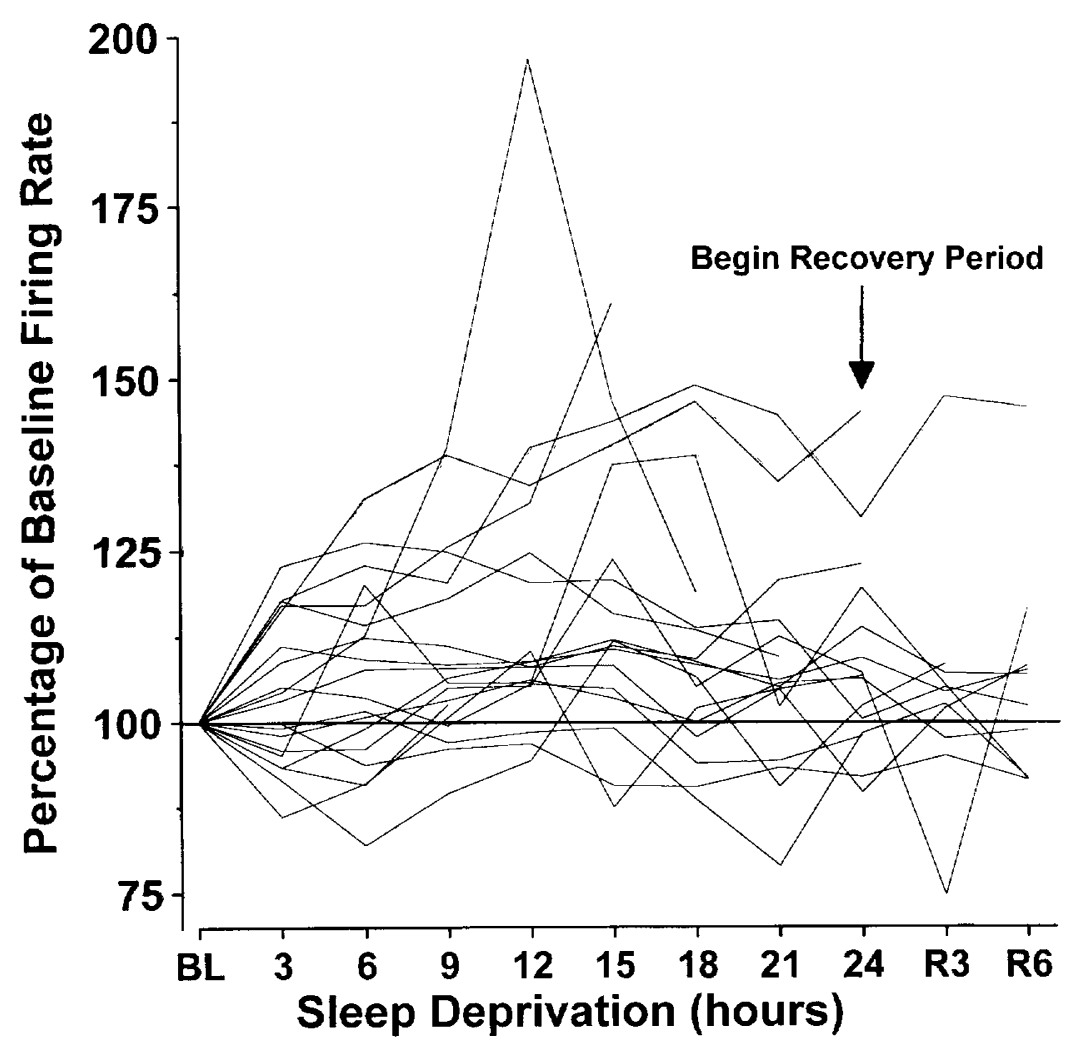

Figure 3. Effect of total sleep deprivation (TSD) and recovery on the activity of individual serotonergic DRN neurons during active waking $(n=19)$. $\mathrm{BL}=$ baseline. During TSD, the activity of individual cells varied from $79 \%$ to $197 \%$ of baseline. 
AW firing rates were also obtained for 11 neurons after 3 and $6 \mathrm{~h}$ of recovery. Six of these cells displayed a significant decrease in firing rate from the 24-h timepoint of sleep deprivation. One cell showed a significant increase in firing rate during the recovery period, and the remaining four cells showed no change in firing rate.

Figure 4 displays the effects of sleep deprivation and subsequent recovery on the activity of serotonergic neurons during QW. A one-way ANOVA revealed a significant overall effect of time on QW firing rates $(p<$ $.05)$, although subsequent post hoc analysis showed that no individual time points during the experiment were significantly different from baseline. The peak increase in mean QW firing rate during the deprivation period was $11.8 \pm 5.9 \%(n=19$ cells $)$ and occurred at 12 $\mathrm{h}$. The response of individual cells to TSD varied. Ten of the 20 neurons showed an increase in QW firing rate during the sleep deprivation period, whereas the remainder of the neurons showed either a decrease $(5 / 20)$ or no change $(5 / 20)$ in firing rate.

The 12 neurons that were recorded during at least one of the two time points in the recovery sleep period showed a variety of responses in their QW firing rates. In total, four of these neurons decreased their QW firing rates during the recovery period, whereas four neurons displayed an increased firing rate. Four neurons did not change their firing rates significantly during the recovery period.

A one-way ANOVA was performed on the normalized (baseline $=100 \%$ ) data to determine whether $\mathrm{AW}$ had a greater effect on firing rate during TSD than QW. All data collected during TSD for these two conditions were included in the analysis. The mean AW firing rate during TSD was significantly elevated above the mean QW firing rate $(p<.05)$.

\section{Response to White Noise Exposure}

The activity of serotonergic DRN neurons was recorded during brief exposure to loud white noise over the course of the sleep deprivation and recovery periods. Figure 5 shows the effect of sleep deprivation and recovery on the unit activity during white noise exposure. The mean baseline firing rate of these neurons during white noise exposure prior to TSD was $3.29 \pm 0.34$ spikes/s $(n=19$ cells). A one-way ANOVA revealed a significant effect of time $(p<.01)$. Post hoc analysis showed that sleep deprivation produced a significant increase in unit activity during white noise exposure at 12 , 18 , and $24 \mathrm{~h}$, but not at $6 \mathrm{~h}$. The peak increase in activity during white noise was $17.7 \pm 6.7 \%(n=17$ cells $)$ and occurred at $18 \mathrm{~h}$. During the recovery period, the activity of serotonergic neurons in response to white noise exposure was not significantly different from baseline.

\section{Response to Phasic Sensory Stimulation}

The effect of TSD and recovery on the responses of serotonergic DRN neurons to repeated presentation of an auditory stimulus (click) was examined. Of the 15 cells

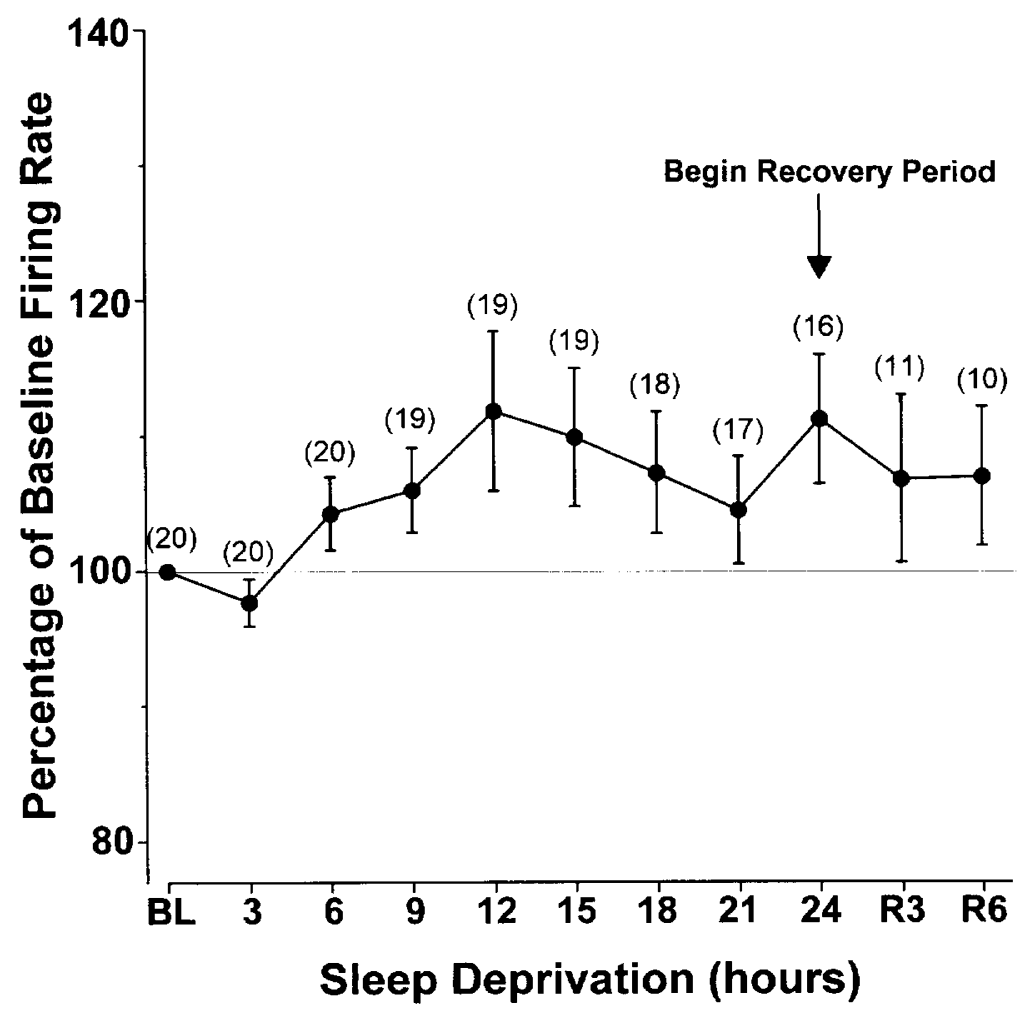

Figure 4. Effect of total sleep deprivation (TSD) and recovery on the activity of serotonergic DRN neurons during quiet waking (QW). Values are means \pm SEM; $n=$ number in parentheses. $\mathrm{BL}=$ baseline. The mean $\mathrm{QW}$ firing rate of these neurons prior to TSD was $3.04 \pm 0.33$ spikes $/ \mathrm{s}$ ( $n=20$ cells). Sleep deprivation had no effect on the QW activity of these neurons during either the deprivation period or the subsequent recovery period. 
studied, eight displayed an excitatory response to click, whereas seven cells showed an inhibitory baseline response. The average latency to excitation or inhibition was approximately $40 \mathrm{~ms}$. The duration of the excitatory response was approximately $50 \mathrm{~ms}$, whereas the duration of the inhibitory response was approximately $200 \mathrm{~ms}$. Of the eight click-excited cells, four showed no change in their response over the course of the sleep deprivation and recovery periods. The remaining four cells showed a decrease in the magnitude of excitatory response. The maximum decrease occurred between 12 $\mathrm{h}$ and $24 \mathrm{~h}$ of TSD. Of these four cells, three were tested during recovery and the excitatory response recovered to baseline levels for all three cells.

Of the seven click-inhibited cells, four showed no change in their response to click over the course of the sleep deprivation and recovery periods. The remaining three cells showed a decrease in the inhibitory response, which was manifested as a decrease in the duration of inhibition, during the deprivation period. The maximum decrease occurred between 12 and $24 \mathrm{~h}$ of TSD. Of these three cells, one was tested during recovery. The inhibitory response of this cell returned to baseline levels.

\section{8-OH-DPAT}

The ability of 8-OH-DPAT to suppress serotonergic DRN unit activity was assessed before and after $24 \mathrm{~h}$ of TSD in eight cells. The mean challenge dose of 8-OH-DPAT used was $10 \pm 6 \mu \mathrm{g} / \mathrm{kg}$. Before TSD, 8-OH-DPAT decreased unit activity by $69.0 \pm 5.4 \%$, relative to preinjection baseline levels. The suppression of activity produced by $8-\mathrm{OH}-\mathrm{DPAT}$ was significantly reduced af- ter $24 \mathrm{~h}$ of TSD $(59.3 \pm 6.9 \% ; p=.05$; paired $t$-test). However, there was no difference in the absolute decreases in firing rate (i.e., spikes/s) produced by $8-\mathrm{OH}$ DPAT before and after TSD ( $2.11 \pm 0.28$ spikes $/ \mathrm{s}$ vs. $2.04 \pm 0.23$ spikes $/ \mathrm{s}$, respectively; $p=0.64$, paired $t$-test). The mean pre-injection firing rate after sleep deprivation was higher than the pre-injection rate before sleep deprivation, although this difference was not statistically significant $(3.54 \pm 0.32$ spikes/s vs. $3.14 \pm 0.44$ spikes/s, respectively; $p=0.20$; paired $t$-test).

The neurons tested with 8-OH-DPAT appeared to be a representative sample of the entire group of cells studied. In addition, six of these cells showed an increase in AW firing rate during TSD, whereas one showed a decrease and one showed no change. Both AW and QW firing rates during the recovery period were unaffected $(p>.05)$ by the administration of 8-OH-DPAT.

\section{Additional Statistical Analyses}

Multiple linear regression analysis revealed that slower-firing neurons, in general, displayed a greater increase in AW discharge rates during TSD than fasterfiring neurons $(p<.05)$. Furthermore, cells that were inhibited by phasic sensory stimulation showed a greater increase in AW firing rates during sleep deprivation than cells showing an excitatory response $(p<.05)$. The time at which the sleep deprivation study began in relation to the light-dark cycle had no significant effect on the peak increase in AW firing rate produced by TSD ( $p>.05$ for all regression models tested). The maximum decrease in firing rate during REM sleep also appeared

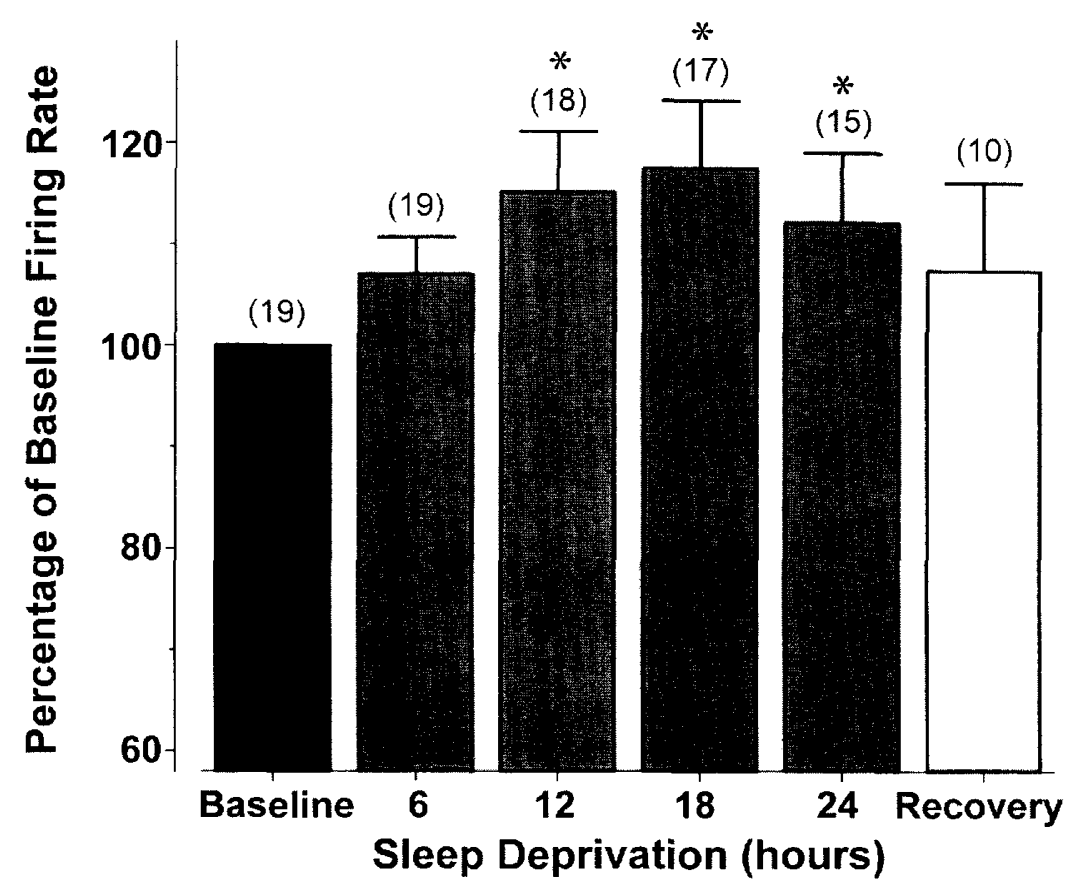

Figure 5. Effect of total sleep deprivation (TSD) and recovery on the activity of serotonergic DRN neurons during exposure to loud white noise. Values are means $\pm \mathrm{SEM} ; n=$ number in parentheses. The mean firing rate of these neurons during white noise exposure prior to TSD was $3.29 \pm 0.34$ spikes $/ \mathrm{s}$ ( $n=19$ cells). Unit activity in response to white noise exposure was significantly increased during the deprivation period, but not during the subsequent recovery period. ${ }^{*} p<.05$ vs. baseline. 
to be unrelated to the peak AW discharge rate during TSD ( $p>.05$ for all regression models tested).

\section{DISCUSSION}

A major finding of the present study is that the firing rate of serotonergic DRN neurons was maintained at or above baseline levels throughout the 24-h TSD period. These results indicate that serotonergic neurons can sustain a relatively high level of activity for prolonged periods of time without showing decrements in firing rate.

The firing rate of serotonergic neurons during $\mathrm{AW}$ was significantly elevated throughout the sleep deprivation period, but not during the subsequent recovery period. QW firing rates were also elevated during TSD, although this effect was small and did not reach statistical significance. The peak increase $(18 \%)$ in mean AW firing rate occurred after $15 \mathrm{~h}$ of TSD. The reason for the decline in unit activity during the latter phase of the sleep deprivation period is not known but may be due to diminished arousal and/or an increase in sleep pressure caused by prolonged wakefulness.

It is unlikely that repeated arousal by the experimenter per se was responsible for the increase in firing rate seen during sleep deprivation, since the frequency and degree of intervention required to prevent sleep varied widely across animals and did not appear to be related to the magnitude of the sleep deprivation effect. Similarly, it is also unlikely that circadian factors had an appreciable influence on neuronal firing rates during TSD, since the activity of serotonergic DRN neurons during AW and QW was found to be stable across the light-dark cycle (Trulson and Jacobs 1983).

Loud white noise was used in the present study as an additional method of eliciting behavioral arousal that did not depend on interaction with the experimenter. This manipulation typically resulted in the cat assuming a crouched and immobile posture, while remaining alert. The mean firing rate of serotonergic neurons during exposure to white noise was significantly increased throughout the sleep deprivation period. The maximum increase in firing rate observed during white noise exposure was nearly identical to the peak increase seen during $\mathrm{AW}$. In addition, the time at which the peak increase in neuronal activity produced by TSD occurred was similar for both white noise exposure and AW (18 h vs. $15 \mathrm{~h}$, respectively). Finally, mean firing rates during both white noise exposure and AW returned to baseline levels during the recovery period. The similarities between these two conditions suggest that the effects of TSD cannot be attributed to either the presence of the experimenter or to differences in the level of experimenter-induced arousal.

During the sleep deprivation period, most cells displayed their largest increase in firing rate during AW and evoked arousal, when serotonergic neuroms generally exhibit their highest level of activity. Since the firing rate of serotonergic neurons is controlled by tonic feedback inhibition mediated by somatodendritic $5-\mathrm{HT}_{1 \mathrm{~A}}$ autoreceptors, an increase in the maximal firing rate would be consistent with a decrease in the sensitivity of these receptors (see below) and a corresponding decrease in feedback inhibition. Furthermore, the fact that slower-firing serotonergic cells displayed greater peak increases in firing rate than faster-firing cells during TSD is consistent with the idea that sleep deprivation causes a desensitization of $5-\mathrm{HT}_{1 \mathrm{~A}}$ autoreceptors, since we have shown that such slower-firing cells are under greater tonic feedback inhibition (Fornal et al. 1994, 1996). However, other mechanisms such as changes in excitatory or inhibitory inputs may be responsible for the observed increase in neuronal activity.

The majority (eight out of 15) of the cells tested in response to phasic sensory stimulation (click) did not display significant changes in their activity over the course of the sleep deprivation and recovery periods. The remaining cells all showed a decrease in the amplitude of either the excitatory or inhibitory response. Previous data from our laboratory suggest that glutamate may mediate excitatory responses of serotonergic DRN neurons to click (Levine and Jacobs 1992), whereas the inhibitory response to click is most likely mediated by gamma-aminobutyric acid (GABA). The attenuated sensory-evoked responses observed for some serotonergic neurons in the present study may underlie the deficits in sensory information processing seen during sleep deprivation in humans.

The ability of 8-OH-DPAT to suppress the activity of serotonergic neurons was determined before and after $\mathrm{TSD}$, in order to assess the sensitivity of $5-\mathrm{HT}_{1 \mathrm{~A}}$ autoreceptors. The maximum percentage decrease in neuronal activity produced by $8-\mathrm{OH}-\mathrm{DPAT}$ was significantly reduced after TSD, suggesting that TSD produces a desensitization of somatodendritic $5-\mathrm{HT}_{1 \mathrm{~A}}$ autoreceptors. However, the absolute decrease in firing rate (spikes/s) produced by 8 -OH-DPAT was not significantly changed after sleep deprivation. The discrepancy between the absolute rate decrease and the relative percentage decrease in neuronal activity produced by $8-\mathrm{OH}$-DPAT may be a consequence of the higher baseline firing rate observed after TSD. Thus, a similar decrease in absolute firing rate from an elevated baseline would manifest itself as a smaller percentage decrease in activity. These data provide preliminary evidence of a decrease in $5-\mathrm{HT}_{1 \mathrm{~A}}$ autoreceptor sensitivity after TSD in the awake cat and are consistent with the results of a recent study in anesthetized rats, which showed that the inhibitory response of serotonergic DRN neurons to an SSRI (citalopram) is diminished after repeated REM sleep deprivation (Maudhuit et al. 1996). It should be noted that the effects of repeated administration of 8-OH-DPAT on serotonergic neuronal activity were not controlled for in these experiments. It 
could be argued that the first injection of 8-OH-DPAT, administered before TSD, might have altered the response of serotonergic neurons to a subsequent injection of 8-OH-DPAT after TSD. However, this is highly unlikely, given the low doses used in this study, and the short duration (approximately $1 \mathrm{~h}$ ) of the 8-OH-DPAT effect. Furthermore, we have previously shown that the inhibitory response of serotonergic neurons to 8-OH-DPAT is not altered by repeated IV administration of the drug (Fornal et al. 1994, 1996).

The maintenance of serotonergic unit activity and not the increase in firing rate observed during prolonged waking may be responsible for the antidepressant effect of TSD. Had the cats been allowed to sleep, the activity of serotonergic neurons would have declined dramatically during each sleep episode. No periods of reduced neuronal activity were observed during TSD. Thus, the pattern of serotonergic unit activity in animals undergoing sleep deprivation is markedly different from that observed in animals allowed to maintain normal sleep-wake cycles. The elimination of periods during which serotonergic neuronal activity is reduced or absent may be a key factor in the antidepressant action of sleep deprivation, since the sustained discharge of serotonergic neurons and corresponding tonic release of 5-HT at postsynaptic receptors would enhance overall 5-HT neurotransmission. The progressive improvement in mood observed in many depressed patients throughout the day ( $\mathrm{Wu}$ and Bunney 1990) may also be due to prolonged activation of the brain serotonergic system. Thus, the antidepressant action of TSD might be viewed as an extension of the therapeutic effects of maintained waking on mood.

Several additional points regarding the present study and the antidepressant action of TSD deserve mention. Depriving cats of sleep for $24 \mathrm{~h}$ may not be equivalent to $24 \mathrm{~h}$ of sleep deprivation in humans. Cats sleep approximately twice as much as humans $(16 \mathrm{~h}$ per day vs. $8 \mathrm{~h}$ per day). Thus, the total amount of sleep loss over a $24-h$ period is considerably larger in cats than in humans. Furthermore, the present study used apparently normal cats, and it is possible that the physiological response to sleep deprivation may differ in depressed subjects.

The present study provides the first direct evidence that TSD alters the electrophysiological activity of brain serotonergic neurons in freely moving animals. Sleep deprivation increased the firing rate of these neurons during AW and arousal, and prevented the suppression of neuronal activity which normally accompanies sleep. This increase in overall serotonergic neuronal activity may play an important role in the antidepressant action of TSD.

\section{REFERENCES}

Blier P, de Montigny C (1994): Current advances and trends in the treatment of depression. Trends Pharmacol Sci 15:220-226

Ferrer A, Artigas F (1994): Effects of single and chronic treatment with tranylcypramine on extracellular serotonin in rat brain. Eur J Pharmacol 263:227-234

Fornal CA, Jacobs BL (1988): Physiological and behavioral correlates of serotonergic single-unit activity. In Osborne NN, Hamon M (eds), Neuronal Serotonin. New York, Wiley, pp 305-345

Fornal CA, Litto WJ, Metzler CW, Marrosu F, Tada K, Jacobs BL (1994): Single-unit responses of serotonergic neurons to $5-\mathrm{HT}_{1 \mathrm{~A}}$ agonist and antagonist drug administration in behaving cats. J Pharmacol Exp Ther 270:1345-1358

Fornal CA, Metzler CW, Gallegos RA, Veasey SC, McCreary AC, Jacobs BL (1996): WAY-100635, a potent and selective


gic neuronal activity in behaving cats: Comparison with (S)-WAY-100135. J Pharmacol Exp Ther 278:752-762

Invernizzi R, Belli S, Samanin R (1992): Citalopram's ability to increase the extracellular concentrations of serotonin in the dorsal raphe prevents the drug's effect in the frontal cortex. Brain Res 584:322-324

Leibenluft E, Wehr TA (1992): Is sleep deprivation useful in the treatment of depression? Am J Psychiatry 149:159-168

Levine ES, Jacobs BL (1992): Neurochemical afferents controlling the activity of serotonergic neurons in the dorsal raphe nucleus: Microiontophoretic studies in the awake cat. J Neurosci 12:4037-4044

Maudhuit C, Hamon M, Adrien J (1996): Effects of chronic treatment with zimelidine and REM sleep deprivation on the regulation of raphe neuronal activity in a rat model of depression. Psychopharmacology 124:267-274

Tobler I, Scherschlicht R (1990): Sleep and EEG slow-wave activity in the domestic cat: Effect of sleep deprivation. Behav Brain Res 37:109-118

Trulson ME, Jacobs BL (1983): Raphe unit activity in freely moving cats: Lack of diurnal variation. Neurosci Lett 36:285-290

Wu JC, Bunney WE (1990): The biological basis of an antidepressant response to sleep deprivation and relapse: Review and hypothesis. Am J Psychiatry 147:14-21 